\title{
Laurent Carroué, 2013, La France - Les mutations des
} systèmes productifs

Paris, A. Colin, Coll.U, 235p.

Aurélien Gack

\section{OpenEdition}

\section{Journals}

Electronic version

URL: http://journals.openedition.org/rge/5174

DOI: $10.4000 /$ rge.5174

ISSN: 2108-6478

\section{Publisher}

Association des géographes de l'Est

Printed version

Date of publication: 15 October 2014

ISSN: 0035-3213

\section{Electronic reference}

Aurélien Gack, «Laurent Carroué, 2013, La France - Les mutations des systèmes productifs 》, Revue

Géographique de l'Est [Online], vol. 54 / 1-2 | 2014, Online since 18 October 2014, connection on 25

September 2020. URL : http://journals.openedition.org/rge/5174; DOI : https://doi.org/10.4000/rge. 5174

This text was automatically generated on 25 September 2020

Tous droits réservés 


\section{Laurent Carroué, 2013, La France - Les mutations des systèmes productifs}

Paris, A. Colin, Coll.U, 235p.

Aurélien Gack

\section{REFERENCES}

Laurent Carroué, 2013, La France - Les mutations des systèmes productifs, Paris, A. Colin, Coll.U, 235p.

1 Le Territoire français, permanences et mutations de Félix Damette et Jacques Scheibling, paru en 1994, dans la suite des travaux du Laboratoire STRATES, était l'une des dernières synthèses générales sur la géographie économique du territoire français. Une actualisation conceptuelle et statistique est permise avec l'ouvrage de L. Carroué qui se veut également une réponse aux interrogations de la géographie économique sur la France, partagées jusqu'au plus haut sommet de l'état, et aussi dans le cadre de la nouvelle question aux Agrégations et CAPES.

2 L'ouvrage est découpé en cinq chapitres. Le premier reprend la notion de système productif dans l'évolution de la pensée de la géographie et de l'économie. Après cette mise au point épistémologique claire sur les outils conceptuels français et anglo-saxons, une analyse multiscalaire, à l'échelle nationale puis urbaine est entreprise dans les deuxième et troisième chapitres. Le quatrième chapitre étudie les dynamiques des sphères de la production, dont la périproduction. Cette dernière est récente, dans cette énonciation, au sein de la Géographie. Enfin, le cinquième chapitre aborde les reproductions sociales et élargies.

3 La méthodologie, à partir des données de l'INSEE est précisée. Ce n'est pas inutile, tant les découpages, à l'instar de celui de la Nomenclature d'Activités Française (NAF) ou des bassins de vie (p.190-191), sont complexes. De nombreux tableaux statistiques et graphiques accompagnent et complètent le texte, ainsi que quelques études de cas à grande échelle, le plus souvent celle du bassin d'emploi. L'analyse des flux de revenus 
et de richesses entre les territoires est particulièrement pertinente, car peu développés jusque-là au sein de la discipline. De nombreuses cartes à l'échelle nationale figurent dans l'ouvrage et permettent souvent, au moins partiellement, de mettre en question les grands leitmotive de la géographie économique de la France, de la célèbre ligne Le Havre-Marseille à l'héliotropisme, seul facteur d'attractivité économique, en passant par la diagonale du vide économiquement atone ou les régions perpétuellement «en reconversion » (Nord-Pas-de-Calais, Lorraine).

4 Cette étude procède également à une synthèse dans les domaines productifs les plus importants en France, comme l'automobile, les systèmes agricoles, l'aérospatiale et l'armement. De même, l'étude du déclin textile et de ses mutations constitue une démonstration des dynamiques de long terme de la géographie productive de la France. Les activités de reproduction sociale ne sont pas oubliées ; le tourisme est ainsi abordé dans la dernière partie, intégré dans une réflexion plus globale sur l'économie résidentielle. L'approche urbaine, surtout par le biais de la métropolisation, incite également à aborder les polarisations croissantes du territoire autour de ces objets géographiques mondialisés que sont les métropoles, ce qui n'est pas sans poser de problèmes au niveau de la cohésion territoriale et sociale du plus grand pays d'Europe occidentale, en superficie.

5 L'analyse régionale est également présente, ce qui souligne l'enjeu d'intégration des potentiels productifs localisés à des tissus productifs et enfin à un système productif de plus en plus mondialisé mais toujours national et européen. L'intégration transfrontalière est ainsi évoquée. Des débats actuels, bien au-delà de la géographie, sont également ouverts. Le coût du capital est ainsi évoqué à la page 63. Les acteurs économiques et sociaux sont intégrés à la réflexion, en particulier dans les recompositions territoriales, soit lancées par l'État, comme les restructurations militaires (p. 195-196), soit par les acteurs privés, avec les espaces des résidences secondaires.

6 L'ouvrage se lit rapidement mais nécessite des connaissances solides pour appréhender toutes les dimensions de la géographie économique développées. Alors que le pays connaît une crise économique et sociale sans précédent depuis l'entre-deux guerres, la lecture géographique de Laurent Carroué souligne les différents défis auxquels l'économie française est confrontée, à la fois en son sein et vis-à-vis des logiques mondialisées. On peut néanmoins regretter l'absence d'une sous-partie consacrée aux réseaux, qui ont un poids croissant, ou bien aux territoires ultramarins, aux dynamiques propres, mais l'ouvrage garde une très forte densité, tant conceptuelle que d'exemples.

7 Au-delà de la sphère géographique, le livre donne un aperçu des raisons de la perte de plus d'un millier d'usines depuis 2008 et les multiples conséquences de la crise sur les recompositions du système productif français. C'est aussi un moyen de susciter, auprès des lecteurs, enseignants, chercheurs et, ou, citoyens, une prise de conscience de l'impact des évolutions économiques récentes sur le territoire français. 


\section{AUTHORS}

\section{AURÉLIEN GACK}

Université de Lorraine 